\title{
Smart surfaces with switchable superoleophilicity and superoleophobicity in aqueous media: toward controllable oil/water separation
}

\author{
Lianbin Zhang, Zhonghai Zhang and Peng Wang
}

\begin{abstract}
Advanced materials with surfaces that have controllable oil wettability when submerged in aqueous media have great potential for various underwater applications. Here we have developed smart surfaces on commonly used materials, including non-woven textiles and polyurethane sponges, which are able to switch between superoleophilicity and superoleophobicity in aqueous media. The smart surfaces are obtained by grafting a block copolymer, comprising blocks of pH-responsive poly(2-vinylpyridine) and oleophilic/hydrophobic polydimethylsiloxane (i.e., P2VP-b-PDMS) on these materials. The P2VP block can alter its wettability and its conformation via protonation and deprotonation in response to the $\mathrm{pH}$ of the aqueous media, which provides controllable and switchable access of oil by the PDMS block, resulting in the switchable surface oil wettability in the aqueous media. On the other hand, the high flexibility of the PDMS block facilitates the reversible switching of the surface oil wettability. As a proof of concept, we also demonstrate that materials functionalized with our smart surfaces can be used for highly controllable oil/water separation processes.
\end{abstract}

NPG Asia Materials (2012) 4, e8; doi:10.1038/am.2012.14; published online 17 February 2012

Keywords: block copolymer; oil/water separation; smart surfaces; wettability

\section{INTRODUCTION}

Recently, the remarkable oil-repellent ability of fish scales, which allows fish to survive in oil-polluted water, has drawn considerable research attention. ${ }^{1}$ Studies reveal that this fascinating ability, which is recognized as underwater superoleophobicity (oil contact angle larger than $150^{\circ}$ ), arises from the combination of hierarchical micro-nanostructures and the hydrophilic chemistry on the surface of the scales. ${ }^{1-3}$ Inspired by fish scales, artificial superoleophobic surfaces in aqueous media have been realized in recent years, and the study of surface oil wettability in aqueous media is emerging as a new research focus. ${ }^{4-7}$ The wettability of oil on a material surface, when submerged in an aqueous environment, has a critical role in many practical applications, such as droplet manipulation in microfluidics, ${ }^{8-10}$ cell and protein adhesion control on surfaces, ${ }^{11-14}$ preparation of electrowetting-based displays ${ }^{15}$ and oil/water separation. ${ }^{16,17}$ It is anticipated that a surface with controlled oil wettability, or more desirably a smart surface that switches its oil wettability in response to external stimuli in aqueous media, would offer great promise in the design and fabrication of intelligent materials for advanced applications. Although considerable efforts have been devoted to controlling oil wettability as well as oil-adhesion properties on material surfaces in aqueous media, ${ }^{5-7}$ smart surfaces with switchable superoleophobicity and superoleophilicity (oil contact angle lower than $10^{\circ}$ ) in aqueous media have not been reported.

Generally, existing methods to prepare superoleophobic surfaces in aqueous media emphasize the use of hydrophilic chemistry in combination with hierarchical surface structures. ${ }^{1,4-6}$ Although the hydrophilic surface chemistry works well for obtaining underwater oleophobicity, it by itself is not possible to switch to oleophilic when immersed in aqueous media, as the hydrophilic surface traps water, and the water-trapped surface repels oil due to the repellency between polar (trapped water in the surface) and non-polar (oil) molecules. To realize oleophilicity in aqueous media, a surface chemistry that is both oleophilic and hydrophobic is generally required. ${ }^{2,18-20}$ For instance, the superhydrophobic (water contact angle larger than $150^{\circ}$ ) and superoleophilic nanowire membrane constructed by Stellacci and co-workers, ${ }^{18}$ exhibited oleophilic property in aqueous media, and can be used for the separation of oil from water. Therefore, to obtain a smart surface with switchable oil wettability in aqueous media, especially between superoleophobicity and superoleophilicity, the chemistry on the surface should be delicately designed such that it comprises both hydrophilic and oleophilic/hydrophobic characteristics, with either characteristic becoming dominantly exposed over the other in response to environmental conditions. 
Block copolymers, consisting of two or more covalently bonded blocks with different physical and chemical properties, represent a promising class of materials suitable for the preparation of smart surfaces. ${ }^{21-25}$ Here we describe a block copolymer-grafting strategy that uses a block copolymer comprising $\mathrm{pH}$-responsive poly(2-vinylpyridine) and oleophilic/hydrophobic polydimethylsiloxane blocks (i.e., P2VP- $b$-PDMS), to functionalize inexpensive and easily available materials, including non-woven textiles and polyurethane sponges, to prepare surfaces with switchable superoleophilicity and superoleophobicity in aqueous media. The P2VP block on the grafted block copolymer can alter its wettability and its conformation via protonation and deprotonation in response to the $\mathrm{pH}$ of the aqueous media, which in turn provides controllable and switchable access of oil by the oleophilic PDMS block on the surface, thus realizing a smart surface with switchable oil wettability in aqueous media. As a proof of concept, we demonstrate the effectiveness and sustainability of our functionalized materials in highly controllable oil/ water separation.

\section{MATERIALS AND METHODS}

\section{Materials}

Poly(2-vinylpyridine- $b$-dimethylsiloxane) block copolymer P5321 (P2VP- $b$ PDMS 16000-b-10000 $\mathrm{g} \mathrm{mol}^{-1}$ ) and carboxy-terminated P2VP P7546 (P2VP-COOH, $10000 \mathrm{~g} \mathrm{~mol}^{-1}$ ) were purchased from Polymer Source, Inc., (Montreal, Quebec, Canada). Sylgard 184 silicone elastomer base and curing agent were purchased from Dow Corning (Midland, MI, USA). Silica nanoparticles (with an average size of $7.0 \mathrm{~nm}$ ), 1,2-bis(triethoxysilyl)ethane, (3-bromopropyl)trimethoxysilane (BPS), 3-glycidoxypropyl trimethoxysilane, tetrahydrofuran (THF), 1,2-dichloroethane (DCE), oil red $\mathrm{O}$ and anhydrous toluene were all purchased from Sigma-Aldrich (St Louis, MO, USA) and used as received. Non-woven textiles (cloth-like wipes made from cellulose and polypropylene blends) were purchased from Workwipes (New Pig Co., Tipton, PA, USA). Water purified in a Milli-Q (Millipore, Billerica, MA, USA) system was used in all experiments.

\section{Experimental procedures}

Preparation of functionalized textile and sponge by the block copolymer grafting. A silica nanoparticle suspension containing $20 \mathrm{ml}$ of $2 \%$ silica nanoparticles $(7.0 \mathrm{~nm}), 0.5 \mathrm{ml}$ concentrated $\mathrm{HCl}$ and $0.5 \mathrm{ml}$ 1,2-bis(triethoxysilyl)ethane was first prepared by mixing under stirring. The non-woven textile was first cleaned by ethanol and water to remove surface impurities and then treated by air plasma for $1 \mathrm{~min}$. Subsequently, the silica nanoparticles were deposited on the textile by a dip-coating process. After drying of the silica-nanoparticledeposited textile in an oven at $60^{\circ} \mathrm{C}$ for $3 \mathrm{~h}$, the textile was washed thoroughly by copious amounts of water and then dried. The silica-nanoparticledeposited textiles were then immersed in a $1 \%$ anhydrous toluene solution of BPS for $12 \mathrm{~h}$ at room temperature to functionalize the textile surface with bromoalkyl groups via silanization. The silanized textile was then rinsed with toluene and ethanol to remove the unreacted silanes, followed by drying with a flow of nitrogen. Finally, the P2VP- $b$-PDMS block copolymers were coated on the silanized textile from a $2 \mathrm{mg} \mathrm{ml}^{-1}$ THF solution by a dip-coating process, and then the textile was put in a vacuum oven at $150^{\circ} \mathrm{C}$ for $12 \mathrm{~h}$ to enable quaternization between the bromoalkyl groups and the pyridine groups of the block copolymers. The ungrafted polymers were removed by washing with copious amounts of THF. Functionalized sponges were prepared using the same procedures.

Preparation of flat surfaces of P2VP-b-PDMS, P2VP and PDMS. P2VP-b$P D M S$-grafted smooth silicon wafer: A cleaned, smooth silicon wafer was first immersed in a $1 \%$ anhydrous toluene solution of BPS for $12 \mathrm{~h}$ at room temperature to functionalize the silicon surface with bromoalkyl groups via silanization. The silanized silicon was then rinsed with toluene and ethanol to remove the unreacted silanes, followed by drying with a flow of nitrogen. The P2VP- $b$-PDMS block copolymer was spin-coated on the silanized silicon surface from a $2 \mathrm{mg} \mathrm{ml}^{-1}$ THF solution, and then the silicon wafer was put in a vacuum oven at $150^{\circ} \mathrm{C}$ for $12 \mathrm{~h}$ to enable quaternization between the bromoalkyl groups and the pyridine groups of the block copolymers. The ungrafted polymers were removed by washing with copious amounts of THF. P2VP-grafted smooth silicon wafer: ${ }^{26}$ A cleaned, smooth silicon wafer was first immersed in a $1 \%$ anhydrous toluene solution of 3-glycidoxypropyl trimethoxysilane for $12 \mathrm{~h}$ at room temperature to functionalize the silicon surface with glycidyl groups. The glycidyl group-grafted silicon wafer was then rinsed with toluene and ethanol to remove the unreacted silanes, followed by drying with a flow of nitrogen. The P2VP-COOH was spin-coated on the silanized silicon surface from a $2 \mathrm{mg} \mathrm{ml}^{-1}$ THF solution, and then the silicon wafer was put in a vacuum oven at $150^{\circ} \mathrm{C}$ for $12 \mathrm{~h}$ to enable a reaction between the glycidyl groups and the end-carboxyl groups of P2VP-COOH. The ungrafted polymers were removed by washing with copious amounts of THF.

Flat PDMS surface: The flat PDMS surface was prepared by mixing the Sylgard 184 silicone elastomer base and curing agent in a 10:1 ratio, degassing and spin-coating this mixture on a silicon wafer, and curing for $12 \mathrm{~h}$ at $60^{\circ} \mathrm{C}$.

Characterization. Air plasma treatment was carried out using a PDC-002 plasma cleaner (Harrick Plasma Company, Ithaca, NY, USA). Scanning electron microscopy images were obtained on an FEI Magellan scanning electron microscope (FEI Company, Hillsboro, OR, USA). Contact angle (CA) measurements were performed with an Attension Theta system (KSV Instruments Ltd, Espoo, Finland) at ambient temperature. Water droplets of $2.0 \mu \mathrm{l}$ were used for the water CA measurements in air. For the underwater oil CA measurements, oil droplets (DCE, ca. $2 \mu \mathrm{l}$ ) were dropped carefully onto the surface of the samples, which were fixed on the bottom of a glass container filled with water of different $\mathrm{pH}$. An average $\mathrm{CA}$ value was obtained by measuring the same sample at three different positions.

Controllable separation of gasoline and water mixtures. A piece of the block copolymer-grafted textile $\left(\sim 2 \times 2 \mathrm{~cm}^{2}\right)$ was fixed between two glass tubes (diameter $\sim 1.5 \mathrm{~cm}$ ), serving as a separation membrane. With the non-acidpretreated textile in place, a mixture of commercial gasoline and $\mathrm{pH} 6.5$ water ( $30 \mathrm{ml}$ gasoline, $10 \mathrm{ml}$ water) was poured into the upper glass tube. The gasoline selectively passed through the textile, whereas the water remained in the upper glass tube. For the acidic water-treated textile, the functionalized textile was first wetted with acidic water with a $\mathrm{pH}$ of 2.0 before the oil/water separation process. Then a mixture of commercial gasoline and $\mathrm{pH} 6.5$ water ( $10 \mathrm{ml}$ gasoline, $30 \mathrm{ml}$ water) was poured into the upper glass tube. Water selectively passed through the textile, whereas gasoline remained in the upper glass tube.

Underwater oil capture and release by the functionalized sponge. DCE of $2 \mathrm{ml}$ stained with oil red $\mathrm{O}$ dye was dropped into a beaker containing water with a $\mathrm{pH}$ of 6.5, and a functionalized sponge was moved to touch the DCE in water. The release of the DCE from the sponge was carried out by putting the oil-loaded sponge into acidic water with a $\mathrm{pH}$ of 2.0 , followed by gentle compression.

\section{RESULTS AND DISCUSSION}

\section{Preparation of block copolymer-grafted non-woven textiles}

Generally, to achieve extreme wettability, a rough surface, especially a hierarchically micro-nanostructured surface, is a prerequisite, which amplifies the surface's intrinsic wetting behavior. ${ }^{27-32}$ Here we first chose a non-woven textile as our substrate, because of its low cost, wide availability, chemical and mechanical robustness, and more importantly, its inherent fiber structure, which provides micro-scale structure. Figure 1a schematically illustrates the block copolymer grafting strategy for preparation of a surface with switchable superoleophilicity and superoleophobicity in aqueous media on a textile substrate. To introduce nanostructures on the textile surface and further increase the surface roughness, silica nanoparticles were first deposited on the textile from a silica suspension, containing silica nanoparticles (diameter $\sim 7.0 \mathrm{~nm}$ ), $\mathrm{HCl}$ and 1,2-bis(triethoxysilyl) ethane. The 1,2-bis(triethoxysilyl)ethane was used as a coupling 
agent, which can react with the hydroxyl groups on the surfaces of silica nanoparticles and the textile to cross-link the silica nanoparticles and improve the adhesion between the silica nanoparticles and the underlying textile fibers. The textile with the deposited silica nanoparticles was then immersed in an anhydrous toluene solution of BPS to functionalize the surface with bromoalkyl groups via silanization,
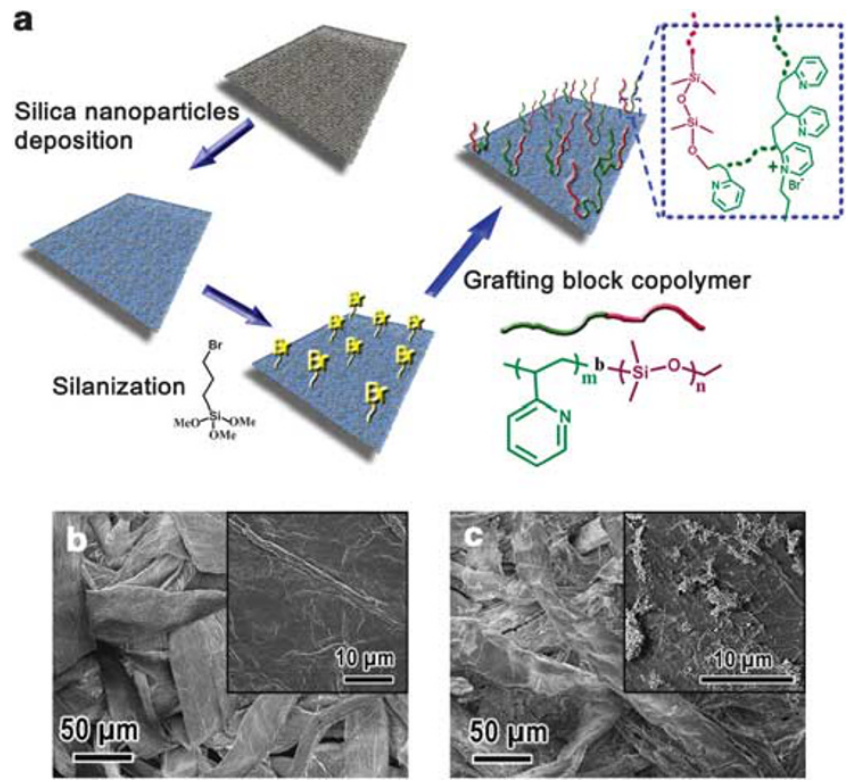

Figure 1 Preparation and characterization of a surface with switchable superoleophilicity and superoleophobicity on a non-woven textile substrate. (a) Schematic showing the preparation strategy for a surface with switchable superoleophilicity and superoleophobicity on a non-woven textile. (b) Scanning electron microscope (SEM) image of the raw textile. Inset: enlarged view of the surface of a single fiber. (c) SEM image of the textile after deposition of silica nanoparticles and block copolymer grafting. Inset: enlarged view of the surface of a single fiber. which served as an intermediate anchoring layer for the block copolymer grafting. Finally, the P2VP- $b$-PDMS block copolymer was dip-coated on the silanized textile from a tetrahydrofuran solution, and the textile was then placed in a vacuum oven at $150^{\circ} \mathrm{C}$ to enable quaternization between the bromoalkyl groups of BPS and the pyridyl groups on the block copolymers, yielding a layer of the grafted block copolymer. Because of the nature of the grafting of the block copolymers via the pyridyl groups in the P2VP blocks, the grafted block copolymer layer can be considered as a mixed polymer brush of P2VP and PDMS chains with broad distribution. ${ }^{33}$

Scanning electron microscopy measurements were carried out to characterize the surface morphologies of the textile before and after these treatments. The raw textile typically had ribbon-like fibers of about $40 \mu \mathrm{m}$ in width and $5 \mu \mathrm{m}$ in thickness with relatively smooth surfaces (Figure 1b). After the deposition of the silica nanoparticles and the grafting of the block copolymer, the basic structure of the textile remained unchanged (Figure 1c). An enlarged view of a textile fiber (Figure 1c, inset) reveals that there were discretely distributed aggregates of the nanoparticles ranging in size from tens of nanometers to several micrometers on the fiber surfaces. These nanoparticle aggregates, along with the inherent micro-sized fibers of the textile, constituted a hierarchically micro-nanostructured surface. Compositional analysis of the textile surface before and after these treatments, which was carried out by X-ray photoelectron spectroscopy measurements, confirmed the successful grafting of the P2VP- $b$ PDMS block copolymer on the surface of the textile by the appearance of a nitrogen signal associated with P2VP (Supplementary Figure S1).

\section{pH-responsive property and switchable oil wettability} of the functionalized textile

As has been reported, $\mathrm{P} 2 \mathrm{VP}$ has a $\mathrm{pH}$-responsive property and it can alter its wettability as well as its conformation via protonation and deprotonation of its pyridyl groups if the $\mathrm{pH}$ changes. ${ }^{34-36}$ The X-ray photoelectron spectroscopy measurement of the functionalized textile indicated that the grafting of the block copolymer on the surface of the textile was achieved via a very small number of 2-vinylpyridine a

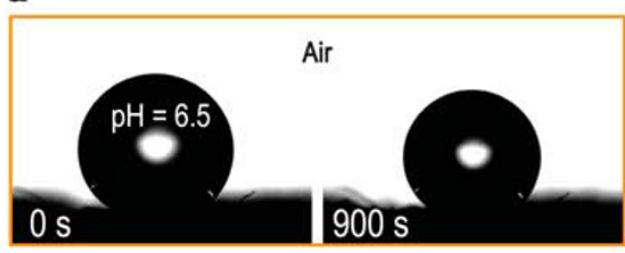

c

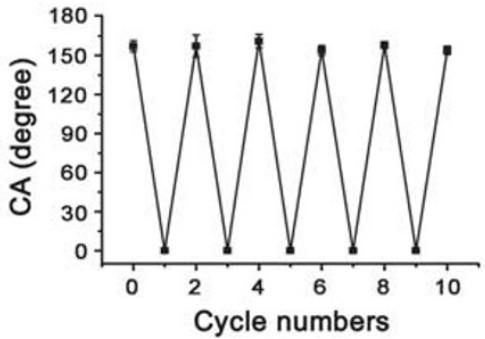

d b

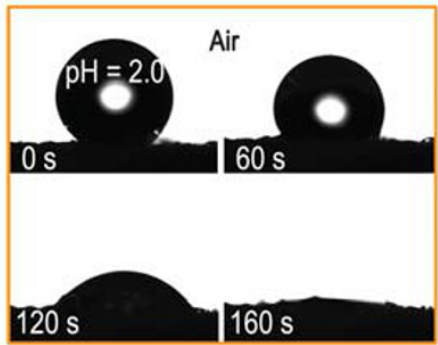

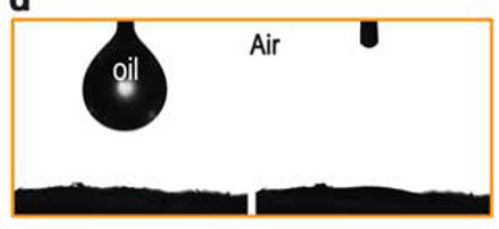

Figure 2 Wettability of the functionalized textile in air. (a) Still images from video $\mathrm{CA}$ measurements for a water droplet $(\mathrm{pH}=6.5)$ applied on the functionalized textile (left) and after $900 \mathrm{~s}$ of exposure (right) in air. (b) Still images from video $\mathrm{CA}$ measurements for a water droplet ( $\mathrm{pH}=2.0$ ) applied on the functionalized textile; the acidic droplet spread over the surface gradually within $160 \mathrm{~s}$, indicating the transition of wettability of the surface from superhydrophobicity to superhydrophilicity. (c) Reversible wettability of the functionalized textile for water droplets with a pH of 6.5 (even cycles) and 2.0 (odd cycles) in air. (d) Still images from video CA measurements of a DCE droplet applied on the functionalized textile surface in air. 
a

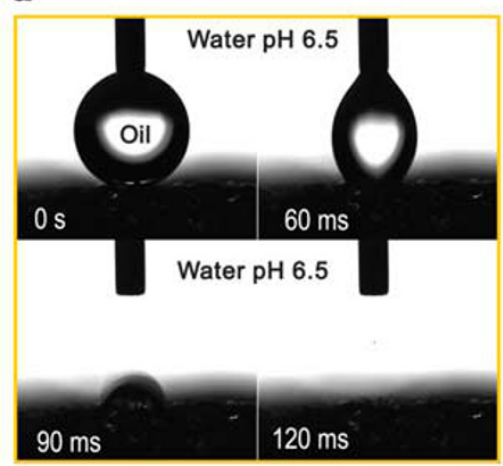

c

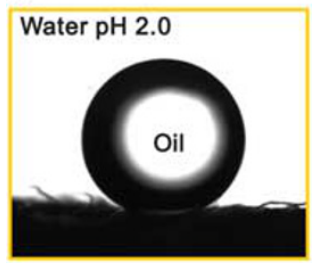

d

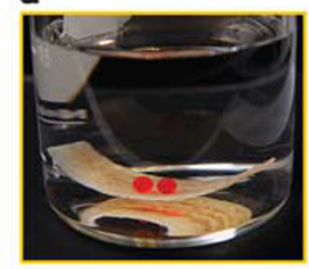

b
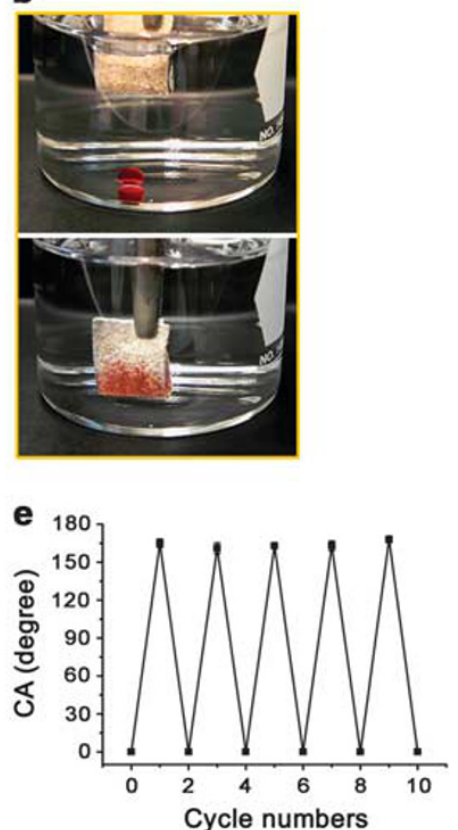

Figure 3 Oil wettability of the functionalized textile in aqueous media. (a) Still images from video CA measurements of a DCE droplet on the functionalized textile surface in water with a pH of 6.5. (b) Snapshots showing the ability of the functionalized textile for the capture of an oil droplet in aqueous media with a $\mathrm{pH}$ of 6.5 . In greater detail, an oil droplet stained with oil red $\mathrm{O}$ was first dropped to the bottom of a beaker containing water with a $\mathrm{pH}$ of 6.5 . Then, a piece of the functionalized textile was lowered into the water to approach the oil droplet. On contact, the textile immediately sucked up the oil droplet, leaving behind no oil residue. (c) Image of a DCE droplet applied on the surface of the textile in water with a pH of 2.0. (d) Photograph of DCE droplets sitting on the functionalized textile in acidic water with a $\mathrm{pH}$ of 2.0. The DEC droplets were stained with oil red 0 for clear observation. (e) Reversible oil wettability of the functionalized textile in aqueous media with a pH of 6.5 (even cycles) and 2.0 (odd cycles).

monomer units, and most of the pyridyl groups on P2VP- $b$-PDMS copolymer remained unchanged during the grafting process, which preserved the $\mathrm{pH}$-responsive property of P2VP (Supplementary Figure S1). CA measurements were first conducted in air to probe the responsive wettability of the functionalized textile surface by using water droplets with different $\mathrm{pH}$ (Supplementary Figure S2). Figure 2a shows that a water droplet with a $\mathrm{pH}$ of 6.5 formed a sphere with a CA of $157.2^{\circ}$ on the textile surface in air. After sitting in ambient air for $15 \mathrm{~min}$, the same water droplet maintained its spherical shape despite its size shrinkage due to evaporation, indicating its stable superhydrophobicity to water with a $\mathrm{pH}$ of 6.5 . As a weak polybase, P2VP has a $\mathrm{p} K_{\mathrm{a}}$ of about 3.6. ${ }^{34}$ When in contact with a water droplet with a $\mathrm{pH}$ of $6.5, \mathrm{P} 2 \mathrm{VP}$ is therefore in the deprotonated state and exhibits a collapsed conformation due to the hydrophobic interaction between the deprotonated P2VP chains. On the other hand, the PDMS block has a desirably low glass transition temperature (Tg) of about $-62{ }^{\circ} \mathrm{C}$ (according to the supplier), and it can be considered as a liquid-like polymer with high flexibility at ambient temperature. ${ }^{26}$ In air, the liquid-like PDMS, which is more hydrophobic than the P2VP, can spontaneously move to the exterior of the grafted copolymer layer and dominantly expose itself to air. ${ }^{37}$ As a consequence, hydrophobic PDMS on the exterior of the grafted block copolymer layer makes the surface hydrophobic, which is further amplified by the hierarchical micro-nanostructures on the surface, resulting in the superhydrophobicity.

However, when a water droplet with a $\mathrm{pH}$ of 2.0 was applied to the same textile in air, it gradually wetted the textile and spread over the surface completely within about $160 \mathrm{~s}$, indicating the superhydrophilic property (water contact angle lower than $10^{\circ}$ ) of the surface to acidic water with a $\mathrm{pH}$ of 2.0 (Figure $2 \mathrm{~b}$ ). When contacting the acidic water, the P2VP chains on the surface become protonated and exhibit an extended conformation because of the electrostatic repulsion among the like charges. They then stretch to the exterior of the grafted block copolymer layer. With the protonated P2VP exposed on the surface, the textile surface acquires hydrophilic property, leading to the wetting with the acidic water. After wetting with the acidic water $(\mathrm{pH}=2.0)$, most of the pyridyl groups on the functionalized textile can be protonated considering the $\mathrm{p} K_{\mathrm{a}}$ (3.6) of P2VP.

Remarkably, the acidic-water wetted textile easily recovered its superhydrophobicity when we rinsed it with water $(\mathrm{pH}$ 6.5) and dried it with nitrogen flow. This treatment can be conducted at room temperature and does not involve the use of extreme heating or organic solvent washing processes. ${ }^{33}$ The reversible cycles can be repeated many times without any change in the responsive property (Figure 2c). The easy reversal of the wetting characteristics of the functionalized textile can be attributed to the highly flexible PDMS of the grafted copolymers. Upon drying, the PDMS, being more hydrophobic than P2VP, can spontaneously move and expose itself on the exterior of the grafted block copolymers again, thereby recovering the superhydrophobicity of the functionalized textile. In addition to hydrophobicity, the dominance of the oleophilic PDMS on the exterior surface endows the functionalized textile with a high affinity to oil. CA measurements using DCE (density, $1.253 \mathrm{~g} \mathrm{~cm}^{-3}$ ) droplets show that, as soon as an oil droplet contacted the functionalized textile surface, it immediately spread out and wetted the textile within $0.05 \mathrm{~s}$, indicating the superoleophilicity of the material in air (Figure 2d).

Having demonstrated the superoleophilicity of the P2VP- $b$-PDMSgrafted textile as well as its switchable wettability to water with 

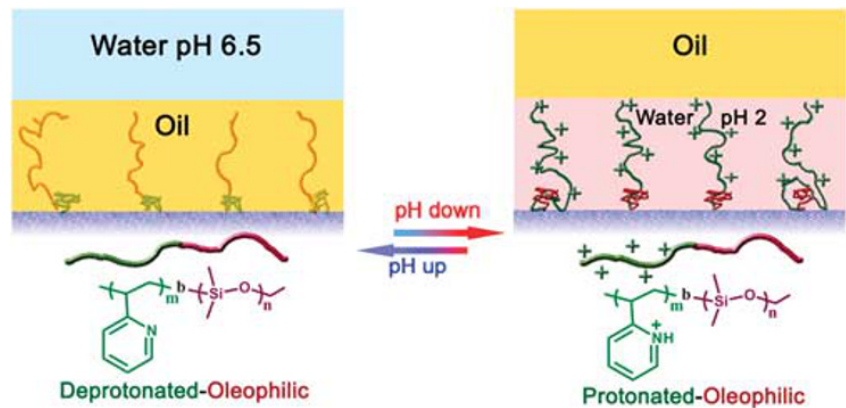

Figure 4 Schematic diagrams for the switchable oil wettability of the P2VP$b$-PDMS-grafted textile. Left, schematic diagram of the oil wettability of the functionalized textile in water with a $\mathrm{pH}$ of 6.5. Right, schematic diagram of the oil wettability of the functionalized textile in water with a $\mathrm{pH}$ of 2.0 .

different $\mathrm{pH}$ in air, we then evaluated the oil wettability of the functionalized textile in aqueous media with different $\mathrm{pH}$. Figure $3 \mathrm{a}$ shows the oil-wetting behavior of the functionalized textile immersed in water with a $\mathrm{pH}$ of 6.5 . It can be observed that as soon as an oil (DCE) droplet touched the surface, it was immediately sucked into the textile within $0.12 \mathrm{~s}$, indicating its superoleophilicity. Because of this superoleophilicity, the block copolymer-grafted textile could be used in underwater oil capture as demonstrated in Figure $3 \mathrm{~b}$. As has been discussed, in water with a $\mathrm{pH}$ of 6.5 , the P2VP is not protonated and maintains its collapsed conformation, which maintains the dominant exposure of the PDMS. The dominantly exposed PDMS prevents the textile from being wetted by water and retains its high affinity to oil, which, assisted by the hierarchical surface structures, leads to superoleophilicity. Upon contact with oil, the PDMS chains preferentially stretch out into the oil because of their oleophilic nature (Figure 4, left panel). Moreover, air could be trapped inside the porous structures of the textile when the superhydrophobic textile is moved from air to water, ${ }^{17,38,39}$ which may also contribute to the superoleophilicity of the functionalized textile in water with a $\mathrm{pH}$ of 6.5. Additionally, it was found that the functionalized textile maintained its superoleophilicity even after $24 \mathrm{~h}$ of immersion in water with a $\mathrm{pH}$ of 6.5 , demonstrating its stable superoleophilicity in such aqueous media.

In contrast, when the functionalized textile was immersed in water with a $\mathrm{pH}$ of 2.0 , the oil wettability of the textile was completely reversed. As shown in Figure 3c, the oil droplet formed a sphere with a CA of $165.3^{\circ}$ on the textile surface in the acidic water, indicating that the textile was superoleophobic. Figure $3 \mathrm{~d}$ is a photograph of oil droplets sitting on the surface of the textile in the acidic water $(\mathrm{pH} 2.0)$. Clearly, the oil did not wet the material. Furthermore, in the acidic water, the oil droplet on such a surface showed a sliding angle less than $5^{\circ}$, which means the oil droplets on the surface could roll off easily, indicating the low oil adhesion of the surface in the acidic water. As discussed previously, when in contact with water with a $\mathrm{pH}$ of 2.0, the textile becomes hydrophilic and wets with the acidic water because of the protonated P2VP chains, which exhibit an extended conformation and are dominantly exposed. The protonated P2VP on the exterior of the grafted block copolymer layer is hydrophilic and traps water, and thus, a water-trapped surface could be formed on top of the oleophilic PDMS, which effectively blocks the access of the oil by the PDMS (Figure 4, right panel). Because of the high repellency between polar (water and protonated P2VP) and non-polar (oil) molecules, the surface exhibits oleophobicity, which is further amplified by the hierarchical structures of the textile, resulting in a superoleophobic surface. After being rinsed with water $(\mathrm{pH} 6.5)$ and dried
Table 1 CA of water and DCE droplets on the different surfaces in air and in water

\begin{tabular}{lcccc}
\hline & \multicolumn{4}{c}{ CA, $\pm 3^{\circ}$} \\
\cline { 2 - 5 } Wetting systems & $\begin{array}{c}\text { Flat silicon with } \\
\text { P2VP-b-PDMS }\end{array}$ & $\begin{array}{c}\text { Flat } \\
\text { PDMS }\end{array}$ & $\begin{array}{c}\text { Flat silicon } \\
\text { with P2VP }\end{array}$ & $\begin{array}{c}\text { Textile with } \\
\text { P2VP-b-PDMS }\end{array}$ \\
\hline Water of pH 6.5 in air & 91.2 & 109.3 & 55.0 & 157.2 \\
Water of pH 2.0 in air & 52.1 & 107.5 & 24.7 & 0 \\
DCE in air & 13.5 & 23.0 & 21.0 & 0 \\
DCE in water of pH 6.5 & 36.5 & 27.4 & 75.5 & 0 \\
DCE in water of pH 2.0 & 137.6 & 25.8 & 132.3 & 165.3
\end{tabular}

Abbreviations: CA, contact angle; DCE, 1,2-dichloroethane; PDMS, polydimethylsiloxane; P2VP, poly(2-vinylpyridine); P2VP-b-PDMS, P2VP-b-dimethylsiloxane).

with nitrogen flow, the superoleophobic textile could easily recover its superoleophilicity in aqueous media with a $\mathrm{pH}$ of 6.5 , and this reversible cycle could be repeated many times without any change in the $\mathrm{pH}$-responsive property of the surface (Figure $3 \mathrm{e}$ ). The reversible cycles of the surface wettability both in air and aqueous media imply that the chemical characteristics of the surface, especially the $\mathrm{pH}$ responsive property of the functionalized textile, are stable during treatment.

As is well known, the wettability of a solid surface is governed by both the chemical composition and the roughness of the surface. ${ }^{27-32}$ To further verify the effect of the $\mathrm{pH}$-responsive surface chemistry on the oil wettability of the functionalized textile, a smooth silicon wafer was used as the substrate onto which the P2VP- $b$-PDMS block copolymer was grafted. This set-up eliminated the influence of surface roughness. Meanwhile, a flat and pure PDMS film and a pure P2VP-grafted smooth silicon wafer were also prepared for comparisons (see Methods). The wettability of these flat surfaces under different conditions was investigated and the results are summarized in Table 1 . In air, the P2VP-b-PDMS-grafted silicon surface was oleophilic and exhibited $\mathrm{pH}$-responsive wettbility to water with different $\mathrm{pH}$, indicating that the surface chemistry changed as a result of deprotonation and protonation of the P2VP. Compared with that of the pure P2VP-grafted flat silicon surface, in air, the much increased CA of the P2VP-b-PDMS-grafted surface to water with a $\mathrm{pH}$ of 6.5 indicates the presence of PDMS on the exterior of the grafted block copolymer layer. Although immersed in water with a $\mathrm{pH}$ of 6.5 , the P2VP- $b$-PDMS-grafted surface also exhibited oleophilic properties with the CA for DCE, similar to that of the flat pure PDMS film in the same water, indicating that the PDMS maintains its dominance on the exterior of the grafted block copolymer layer. However, when immersed in water with a $\mathrm{pH}$ of 2.0, the P2VP- $b$-PDMS-grafted surface became oleophobic with the CA for DCE, similar to that of the pure P2VP-grafted surface in acidic water $(\mathrm{pH}=2.0)$. Given that the flat pure PDMS film had a constant $\mathrm{CA}$ for DCE in water with different $\mathrm{pH}$, the distinct change of the oil wettability on the P2VP- $b$-PDMS-grafted surface implies the dominant exposure of the protonated $\mathrm{P} 2 \mathrm{VP}$ on the exterior of block copolymer layer in the acidic water, which blocks the access of oil by the oleophilic PDMS. The oil droplet actually sits on a watertrapped surface formed by extended and hydrophilic P2VP in the protonated state in the acidic water. Therefore, our results demonstrated that the grafting of P2VP- $b$-PDMS endowed the surfaces with a unique $\mathrm{pH}$-responsive chemistry, which induced switchable oil wettability in aqueous media with different $\mathrm{pH}$. Considering that the surface chemical composition of the P2VP-b-PDMS-grafted smooth 
a

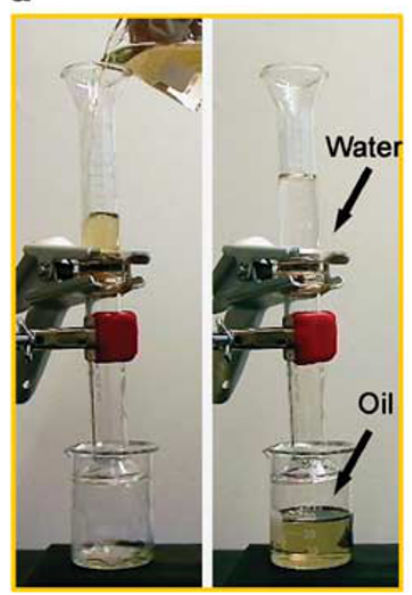

b

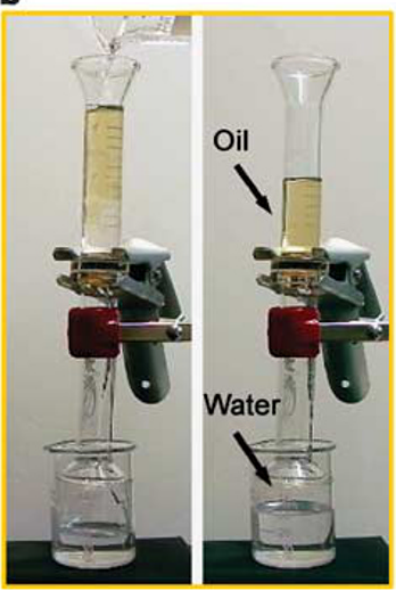

Figure 5 Controllable oil/water separation using the functionalized textile. (a) The functionalized textile was fixed between two glass tubes as the separation membrane. A mixture of gasoline and water was poured into the upper glass tube. The gasoline selectively passed through the textile, whereas the water remained in the upper glass tube (right panel). (b) The functionalized textile was first wetted with acidic water $(\mathrm{pH}=2.0)$ before the water/oil separation process. Water selectively passed through the textile, whereas gasoline remained in the upper glass tube (right panel).

silicon and the functionalized textile is identical, it can be confirmed that the rough surface structure of the textile amplifies the oil-wetting behavior, leading to switchable superoleophilicity and superoleophobicity in aqueous media.

\section{Applications of the functionalized materials to controllable oil/water separation}

The special surface property of switchable superoleophobicity and superoleophilicity is expected to be very useful in materials used in many applications. As a proof of concept, we demonstrated that the functionalized textile could be used for controllable oil/water separation. In our study, the functionalized textile was used as a separation membrane due to its micro-textured and porous structure, which allows the easy and free passage of a wetting liquid. As shown in Figure 5, the functionalized textile was fixed between two glass tubes and a mixture of commercial gasoline and water with a $\mathrm{pH}$ of 6.5 was poured into the upper glass tube (Supplementary Movie S1). Because of the superoleophilicity and superhydrophobicity of the functionalized textile, the gasoline quickly passed through the textile membrane, but the water did not. It was retained on top of the textile membrane in the upper glass tube (Figure 5a). However, when the textile was first simply wetted by acidic water with a $\mathrm{pH}$ of 2.0 without subsequent drying and then used under the same
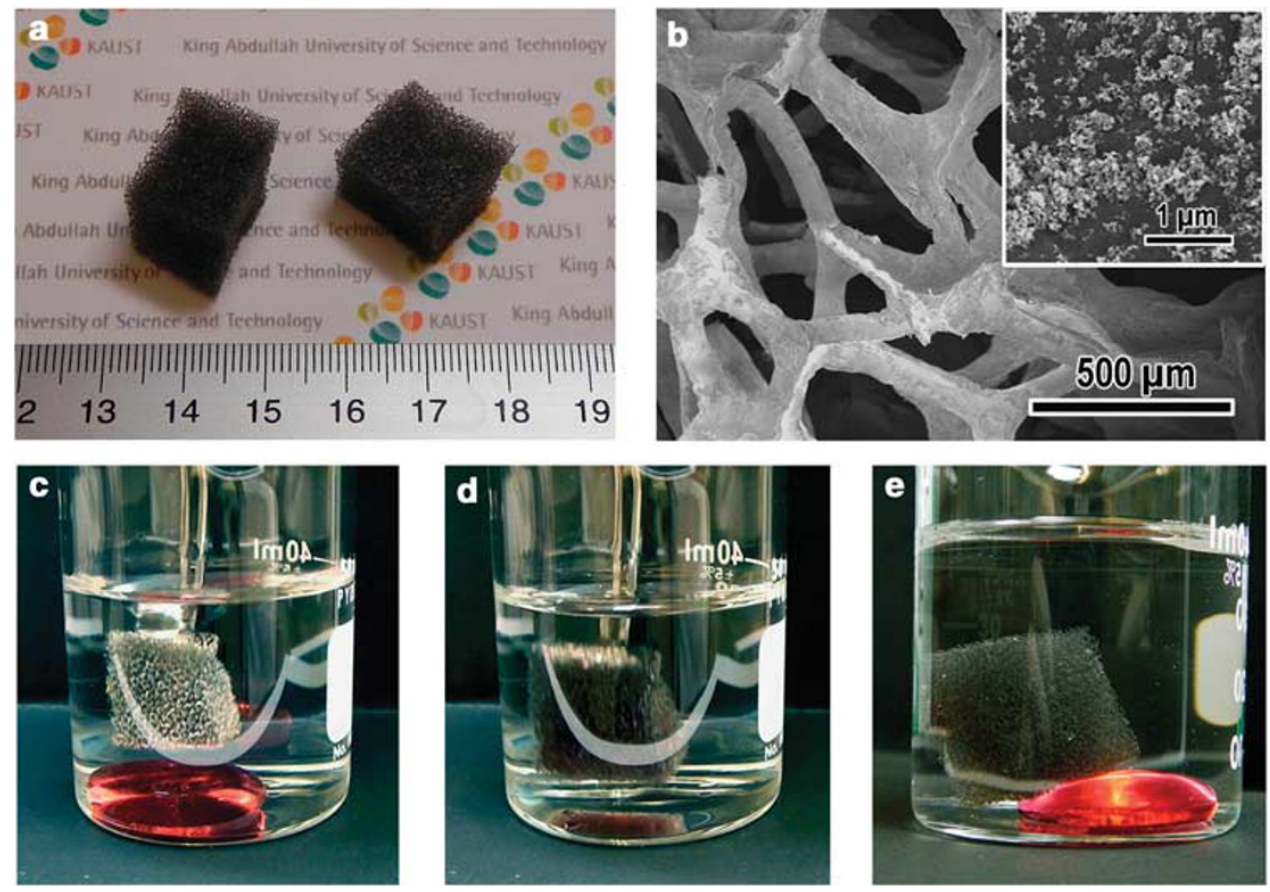

Figure 6 Functionalized polyurethane sponge prepared using the block copolymer grafting strategy and its application to reversible oil capture and release in aqueous media. (a) Digital photograph of the functionalized sponges. (b) Scanning electron microscope (SEM) image of the functionalized sponge. Inset shows the enlarged view of the wall of the sponge. (c and d) Snapshots showing the adsorption of a DCE droplet of about $2 \mathrm{ml}$ (stained with oil red 0 dye) by the functionalized sponge in water with a $\mathrm{pH}$ of 6.5 . In greater detail, $2 \mathrm{ml}$ of DCE stained with oil red $\mathrm{O}$ was first dropped to the bottom of a beaker containing water with a $\mathrm{pH}$ of 6.5. Then, a piece of the functionalized sponge was lowered into the water to approach the oil droplets. On contact, the DCE was quickly sucked up by the functionalized sponge, leaving no oil residue. (e) Release of the captured DCE from the same sponge in acidic water with a pH of 2.0 after compression. After release of the oil in the water with a pH of 2.0, the functionalized sponge can sit on the oil droplet without any adsorption. 
conditions, the opposite separation was realized (Figure 5b), with water passing through the membrane this time. Gravity was the only available force and no external force was used during the separation processes. The ease with which the permeability of the textile for oil or water could be selectively switched on demand makes it possible to use the same material for different separation purposes. To the best of our knowledge, such a versatile material in terms of selective oil/water separation has not been reported before.

The present block copolymer grafting strategy is versatile and could be applied to many common substrates to create functionalized materials with switchable oil wettability capabilities in aqueous media. For instance, by using our block copolymer grafting strategy, we functionalized commonly used polyurethane sponges with switchable superoleophilicity and superoleophobicity in aqueous media (Figures $6 a$ and $b$, and Supplementary Figure S3). The functionalized sponges could then be used for reversible oil capture and release in aqueous media. As illustrated in Figures $6 c-e$, when a functionalized sponge was held to approach DCE in water with a $\mathrm{pH}$ of 6.5 , the DCE was quickly sucked up by the sponge upon contact without any residue left behind (Figures $6 \mathrm{c}$ and $\mathrm{d}$, and Supplementary Movie S2). Moreover, the absorbed oil could be easily released from the sponge by putting the oil-loaded sponge into acidic water $(\mathrm{pH} 2.0)$ followed by gentle compression. As shown in Figure 6e, in the acidic water $(\mathrm{pH} \mathrm{2.0)}$, the water occupied the pores of the functionalized sponge and the sponge repelled the oil. After washing with water ( $\mathrm{pH}$ 6.5) and drying with nitrogen flow, the functionalized sponge recovered its superoleophilicity in aqueous media with a $\mathrm{pH}$ of 6.5 , and thus, could be used again for selective oil removal from water. In our study, the maximum amount of oil (i.e., DCE) that the functionalized sponge could absorb was measured to be 50 times the sponge's weight. Thus, our functionalized sponges are expected to become sustainable and highly cost-effective materials for oil-spill cleanup as the materials can be regenerated and recycled many times.

\section{CONCLUSIONS}

In conclusion, we have demonstrated that smart surfaces with switchable superoleophilicity and superoleophobicity in aqueous media can be realized on commonly used materials, including textiles and sponges, by grafting a block copolymer comprising $\mathrm{pH}$-responsive P2VP and oleophilic/hydrophobic PDMS blocks on these materials. We showed that the functionalized materials with the switchable superoleophilicity and superoleophobicity can be used in highly controllable oil/water separation. We believe that this study will open a new avenue to design and prepare functionalized interfacial materials for underwater applications.

\section{CONFLICT OF INTEREST}

The authors declare no conflict of interest.

\section{ACKNOWLEDGEMENTS}

This work was fully supported by the KAUST baseline fund. We are extremely grateful to Professor Jean MJ Fréchet for helpful discussions. Many thanks are also given to Dr Virginia A Unkefer for help in manuscript preparation.

Author contributions: LBZ and PW conceived the project and planned the experiments. LBZ prepared samples and carried out the wettability characterizations. ZHZ carried out electron microscopy studies. LBZ and PW analyzed the data and wrote the paper. All authors discussed the results and commented on the paper.
1 Liu, M. J., Wang, S. T., Wei, Z. X., Song, Y. L. \& Jiang, L. Bioinspired design of a superoleophobic and low adhesive water/solid Interface. Adv. Mater. 21, 665-669 (2009).

2 Jung, Y. C. \& Bhushan, B. Wetting behavior of water and oil droplets in three-phase interfaces for hydrophobicity/philicity and oleophobicity/philicity. Langmuir 25, 14165-14173 (2009).

3 Nosonovsky, M. \& Bhushan, B. Multiscale effects and capillary interactions in functional biomimetic surfaces for energy conversion and green engineering. Phil. Trans. $R$. Soc. A 367, 1511-1539 (2009).

4 Lin, L., Liu, M. J., Chen, L., Chen, P. P., Ma, J., Han, D. \& Jiang, L. Bio-inspired hierarchical macromolecule-nanoclay hydrogels for robust underwater superoleophobicity. Adv. Mater. 22, 4826-4830 (2010).

5 Chen, L., Liu, M. J., Lin, L., Zhang, T., Ma, J., Song, Y. L. \& Jiang, L. Thermalresponsive hydrogel surface: tunable wettability and adhesion to oil at the water/solid interface. Soft Matter 6, 2708-2712 (2010).

6 Huang, Y., Liu, M. J., Wang, J. X., Zhou, J. M., Wang, L. B., Song, Y. L. \& Jiang, L. Controllable underwater oil-adhesion-interface films assembled from nonspherical particles. Adv. Funct. Mater. 21, 4436-4461 (2011).

7 Liu, M. J., Nie, F. Q., Wei, Z. X., Song, Y. L. \& Jiang, L. In situ electrochemical switching of wetting state of oil droplet on conducting polymer films. Langmuir 26, 3993-3997 (2010).

8 Ionov, L., Houbenov, N., Sidorenko, A., Stamm, M. \& Minko, S. Smart microfluidic channels. Adv. Funct. Mater. 16, 1153-1160 (2006).

9 Truman, P., Uhlmann, P., Frenzel, R. \& Stamm, M. A. Stack of functional nanolayers for simultaneous emulsion separation and sensing. Adv. Mater. 21, 3601-3604 (2009).

10 Schneider, M., Willaime, H., Tran, H. Y., Rezgui, F. \& Tabeling, P. Wettability patterning by UV-initiated graft polymerization of poly(acrylic acid) in closed microfluidic systems of complex geometry. Anal. Chem. 82, 8848-8855 (2010).

11 Cho, E. C., Kim, D. H. \& Cho, K. Contact angles of oils on solid substrates in aqueous media: correlation with AFM data on protein adhesion. Langmuir 24, 9974-9978 (2008).

12 Chen, L., Liu, M. J., Bai, H., Chen., P. P., Xia, F., Han, D. \& Jiang, L. Antiplatelet and thermally responsive poly( $\mathrm{N}$-isopropylacrylamide) surface with nanoscale topography. J. Am. Chem. Soc. 131, 10467-10472 (2009).

13 Sheparovych, R., Motornov, M. \& Minko, S. Low adhesive surfaces that adapt to changing environments. Adv. Mater. 21, 1840-1844 (2009).

14 Wischerhoff, E., Uhlig, K., Lankenau, A., Börner, H. G., Laschewsky, A., Duschl, C. \& Lutz, J.- F. Controlled cell adhesion on PEG-based switchable surfaces. Angew. Chem. Int. Ed. 47, 5666-5668 (2008).

15 Hayes, R. A. \& Feenstra, B. J. Video-speed electronic paper based on electrowetting. Nature 425, 383-385 (2003).

16 Xue, Z. X., Wang, S. T., Lin, L., Chen, L., Liu, M. J., Feng, L. \& Jiang, L. A novel superhydrophilic and underwater superoleophobic hydrogel-coated mesh for oil/water separation. Adv. Mater. 23, 4270-4273 (2011)

17 Jin, M. H., Wang, J., Yao, X., Liao, M. Y., Zhao, Y. \& Jiang, L. Underwater oil capture by a three-dimensional network architectured organosilane surface. Adv. Mater. 23, 2861-2864 (2011).

18 Yuan, J. K., Liu, X. G., Akbulut, O., Hu, J. Q., Suib, S. L., Kong, J. \& Stellacci, F. Superwetting nanowire membranes for selective absorption. Nat. Nanotech. 3, 332-336 (2008).

19 Zhang, Y. L., Wei, S., Liu, F. J., Du, Y. C., Liu, S., Ji, Y. Y., Yokoi, T., Tatsumi, T. \& Xiao, F. S. Superhydrophobic nanoporous polymers as efficient adsorbents for organic compounds. Nano. Today 4, 135-142 (2009).

20 Cheng, M. J., Gao, Y. F., Guo, X. P., Shi, Z. Y., Chen, J. F. \& Shi, F. A functionally integrated device for effective and facile oil spill cleanup. Langmuir 27, 7371-7375 (2011).

21 Rodríguez-Hernández, J., Chécot, F., Gnanou, Y. \& Lecommandoux, S. Toward 'smart' nano-objects by self-assembly of block copolymers in solution. Prog. Polym. Sci. 30, 691-724 (2005).

22 Blanazs, A., Armes, S. P. \& Ryan, A. J. Self-assembled block copolymer aggregates: from micelles to vesicles and their biological applications. Macromol. Rapid Comm. 30, 267-277 (2009).

23 Luzinov, I., Minko, S. \& Tsukruk, V. V. Responsive brush layers: from tailored gradients to reversibly assembled nanoparticles. Soft Matter 4, 714-725 (2008).

24 Stuart, M. A. C., Huck, W. T. S., Genzer, J., Müller, M., Ober, C., Stamm, M., Sukhorukov, G. B., Szleifer, I., Tsukruk, V. V., Urban, M., Winnik, F., Zauscher, S., Luzinov, I. \& Minko, S. Emerging applications of stimuli-responsive polymer materials. Nat. Mater. 9, 101-113 (2010).

25 Kumar, S., Dory, Y. L., Lepage, M. \& Zhao, Y. Surface-grafted stimuli-responsive block copolymer brushes for the thermo-, photo- and $\mathrm{pH}$-sensitive release of dye molecules. Macromolecules 44, 7385-7393 (2011).

26 Motornov, M., Sheparovych, R., Katz, E. \& Minko, S. Chemical gating with nanostructured responsive polymer brushes: mixed brush versus homopolymer brush. ACS Nano. 2, 41-52 (2008).

27 Callies, M. \& Quéré, D. On water repellency. Soft Matter 1, 55-61 (2005).

28 Tuteja, A., Choi, W., Ma, M. L., Mabry, J. M., Mazzella, S. A., Rutledge, G. C., McKinley, G. H. \& Cohen, R. E. Designing superoleophobic surfaces. Science 318, 1618-1622 (2007).

29 Quéré, D. Wetting and roughness. Annu. Rev. Mater. Res. 38, 71-99 (2008).

30 Gao, L. C., McCarthy, T. J. \& Zhang, X. Wetting and superhydrophobicity. Langmuir 25, $14100-14104$ (2009).

31 Liu, K. S., Yao, X. \& Jiang, L. Recent developments in bio-inspired special wettability. Chem. Soc. Rev. 39, 3240-3255 (2010). 
32 Li, Y., Li, L. \& Sun, J. Q. Bioinspired self-healing superhydrophobic coatings. Angew. Chem. Int. Ed. 49, 6129-6133 (2010).

33 Motornov, M., Sheparovych, R., Lupitskyy, R., MacWilliams, E., Hoy, O., Luzinov, I. \& Minko, S. Stimuli-responsive colloidal systems from mixed brush-coated nanoparticles. Adv. Funct. Mater. 17, 2307-2314 (2007).

34 Chang, S., Singamaneni, S., Kharlampieva, E., Young, S. L. \& Tsukruk, V. V. Responsive hybrid nanotubes composed of block copolymer and gold nanoparticles. Macromolecules 42, 5781-5785 (2009).

35 Fujii, S., Kameyama, S., Armes, S. P., Dupin, D., Suzaki, M. \& Nakamura, Y. pHresponsive liquid marbles stabilized with poly(2-vinylpyridine) particles. Soft Matter $\mathbf{6}$, 635-640 (2010).

36 Kim, E. J., Kang, C. J., Baek, H. Y., Hwang, K. Y., Kwak, D. W., Lee, E. K., Kang, Y. J. \& Thomas, E. L. Control of optical hysteresis in block copolymer photonic gels: a step towards wet photonic memory films. Adv. Funct. Mater. 20, 1728-1732 (2010).
37 Jeong, J. W., Park, W. I., Kim, M.- J., Ross, C. A. \& Jung, Y. S. Highly tunable selfassembled nanostructures from a poly(2-vinylpyridine- $b$-dimethylsiloxane) block copoIymer. Nano. Lett. 11, 4095-4101 (2011).

38 Bobji, M. S., Kumar, S. V., Asthana, A. \& Govardhan, R. N. Underwater sustainability of the 'Cassie' state of wetting. Langmuir 25, 12120-12126 (2009).

39 Poetes, R., Holtzmann, K., Franze, K. \& Steiner, U. Metastable underwater superhydrophobicity. Phys. Rev. Lett. 105, 166104 (2010).

(c) This work is licensed under the Creative Commons Attribution-NonCommercial-Share Alike 3.0 Unported License. To view a copy of this license, visit http://creativecommons. org/licenses/by-nc-sa/3.0/

Supplementary Information accompanies the paper on NPG Asia Materials website (http://www.nature.com/am) 